4 Lönnroth K, Castro K, Chakaya JM, et al. Tuberculosis control and elimination 2010-50: cure, care, and social development. Lancet 2010; 375: $1814-29$.

5 Tanimura T, Jaramillo E, Weil D, Raviglione M, Lönnroth K. Financial burden for tuberculosis patients in low- and middle-income countries-a systematic review. Eur Respir J 2014; 43: 1763-75.

6 WHO. Global strategy and targets for tuberculosis prevention, care and control after 2015. A67/11. Geneva: World Health Organization, 2014.

7 Lönnroth K, Glaziou P, Weil D, Floyd K, Uplekar M, Raviglione M. Monitoring universal health coverage and social protection in the context of tuberculosis care and prevention. PLoS Med 11: e1001693.
8 Boccia D, Hargreaves J, Lönnroth K, et al. Cash transfer and microfinance interventions for tuberculosis control: review of the impact evidence and policy implications. Int J Tuberc Lung Dis 2011; 15: S37-49.

9 Rose G. Sick individuals and sick populations. Int J Epidem 1985; 14: 32-38.

10 WHO. Towards tuberculosis elimination in low-incidence countries: a framework. Geneva: World Health Organization, 2014

11 Lönnroth K, Migliori GB, Raviglione M. Towards tuberculosis elimination in low-incidence countries-reflections from a global consultation. Ann Intern Med (in press).

\title{
@ Ebola in west Africa: from disease outbreak to humanitarian crisis
}

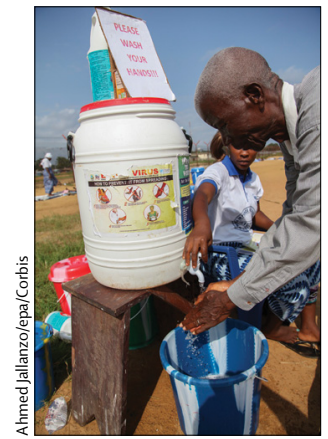

Published Online October 2, 2014 http://dx.doi.org/10.1016/ S1473-3099(14)70956-9
The epidemic of Ebola haemorrhagic fever in west Africa is the 25th known outbreak since $1976,{ }^{1}$ but is fundamentally different from all previous cases. Besides the fact that it is the first Ebola outbreak in west Africa, it is the largest and longest Ebola epidemic, and the first to involve three entire countries and capital cities, with around 5000 cases and 2500 deaths so far. ${ }^{2}$ How could it get to this point? The answer is the synergy of several factors that created a perfect storm: ${ }^{3}$ a context of decades of civil war leading to a low level of trust in authorities, even when these are working hard to reconstruct the country; dysfunctional health services with a major scarcity of health workers, especially in Liberia and Sierra Leone (another consequence of armed conflict); strong traditional beliefs in disease causation and even denial of the virus' existence; high-risk traditional funeral practices that amplify transmission, in addition to more recent healing practices in some churches where the bodies of patients with Ebola are touched; a slow and inadequate national and international response (although this is now changing); and high population mobility across borders-something that has not happened around previous outbreaks in central Africa.

With whole countries affected, and an unprecedented number of infected and exposed people, the epidemic will be far more difficult to control than all previous outbreaks, which typically occurred around hospitals in rural areas or small towns. The sheer scale of the outbreak has, in itself, fundamentally changed the epidemiology, making the disease control prospects bleak. Overwhelmed treatment and isolation facilities turn patients away, so they remain in the community for longer, resulting in even more cases. So the main difference between this and previous epidemics is merely scale, not a mutated Ebola virus.

With a doubling time of around 2 weeks in Monrovia, Liberia, ${ }^{2}$ the window of opportunity for control becomes ever narrower. The risk is also very real that other neighbouring countries, such as Côte d'Ivoire, will soon be affected, although Senegal seems to have effectively averted an outbreak following the introduction of Ebola infection to the country by a Guinean man. ${ }^{4}$

The west African epidemic has profoundly changed how we view Ebola virus infection, which has transformed from a rare event in central Africa into a major public health and destabilising humanitarian crisis. This situation was totally unexpected, but perhaps it should not have been. A recent analysis ${ }^{5}$ of the first epidemic in 1976 in Yambuku (Democratic Republic of the Congo) suggests that even here, reproduction number, RO, in the community (not just the hospital) was probably greater than one. This finding implies that a large-scale community outbreak was always possible given sufficient time. The recent epidemic has confirmed that RO for Ebola in African communities seems to be substantially higher than one, which implies that even if we curtail this outbreak, we should expect another major one to occur eventually. ${ }^{5}$

This shift in thinking has major implications for what is needed to stop the epidemic. Caring for patients, isolating infectious individuals, protecting health staff and other caregivers, safe burials, and contact tracing are the keystones of epidemic control. However, in the face of a massive epidemic with tens of thousands of contacts, implementation challenges of operating 
at a large enough scale are formidable. Additionally, the effectiveness of such approaches might be greatly reduced and insufficient to completely eliminate virus transmission. Thanks to the very professional, and often heroic, work of Médecins sans Frontieres and local partners, we know how to care for patients in such resource-poor settings and how to avoid Ebola transmission in hospital settings-a key component of the response, given that around 150 health-care workers have already died in this epidemic. What is less clear is how to significantly reduce transmission in the community, including how to manage holding centres and those who have fever, and what the incentives are to change risky traditional burial practices. This is a research agenda that could potentially have an immediate effect on epidemic control.

Trials of experimental therapies, starting with the use of hyperimmune plasma or serum from convalescent patients, will begin soon, and should lead to much reduced mortality and transmission. The scale of the epidemic might have reached the point that it can no longer be controlled without a vaccine, and Ebola could become endemic in large communities. Whether the vaccine trials starting now can still make a difference in the present epidemic is unlikely, unless it continues for a very long time or Ebola becomes endemic in west Africa.

Now is also the time to address the huge economic and societal havoc that Ebola is causing in the region, while planning for rebuilding of health systems and disease surveillance.

For the first time in a period of peace, the military are engaging in epidemic control to a major degree. Other nations should follow the USA's initiative in Liberia. The Ebola crisis needs every institution to play its part since the effort is still not commensurate with the actual and potential threat to people in west Africa and possibly elsewhere. That includes also academic institutions and the UK National Health Service, as now made possible following a letter by the Chief Medical Officer inviting staff to volunteer in west Africa.

Other outbreaks of Ebola will occur, as the populations exposed to the probable virus reservoir are expanding in Africa ${ }^{6}$ and the potential for a large epidemic remains where fertile ground for it exists. This has major implications for the future. A large epidemic can-and will-happen again, unless we always remain extremely vigilant, respond promptly, and have more to offer than isolation and quarantine.

*Peter Piot, Jean-Jacques Muyembe, W John Edmunds London School of Hygiene \& Tropical Medicine, Keppel Street, London, WC1E 7HT, UK (PP, WJE); Institut National de Recherche Biomédicale, Kinshasa, Democratic Republic of the Congo (J-J M) peter.piot@Ishtm.ac.uk

We declare no competing interests.

1 International Commission. Ebola haemorrhagic fever in Zaire, 1976 Bull World Health Organ 1978; 56: 271-93.

2 WHO Ebola Response Team. Ebola virus disease in West Africa - the first nine months of the epidemic and forward projections. N Engl J Med 2014 published online Sept 23. DOI:10.1056/NEJMMoa1411100.

3 Piot P. Ebola's perfect storm. Science 2014; 345: 1221.

4 WHO: Ebola Response Roadmap Situation Report. 24 September 2014 http://apps.who.int/iris/bitstream/10665/134771/1/ roadmapsitrep_24Sept2014_eng.pdf?ua=1 (accessed 29 Sept, 2014).

5 Camacho A, Kucharski AJ, Funk S, Breman J, Piot P, Edmunds J. Potential for large outbreaks of Ebola virus disease. Epidemics (in press)

6 Pigott DM, Golding N, Mylne A, et al. Mapping the zoonotic niche of Ebola virus in Africa. eLife 2014; 3: e04395.

\section{Digital surveillance for enhanced detection and response to outbreaks}

In 2014, the Director-General of WHO declared two public health emergencies of international concern: poliomyelitis and Ebola virus disease. These declarations have refocused attention on the use of the International Health Regulations (2005) to identify public health emergencies and to the challenges that state parties face in their assessment. The International Health Regulations (2005) is the principal document that governs international surveillance and response to global public health risks and emergencies. ${ }^{1}$ The document aims to strengthen state parties' abilities to monitor, detect, assess, and report public health hazards in a way that does not adversely affect crossborder travel and trade. ${ }^{2}$ The regulations contain a decision-making matrix that guides state parties' assessment and notification of potential public health

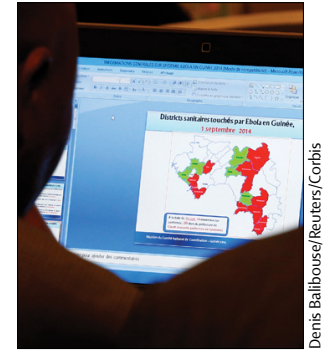

